\title{
ON THE REPRESENTATIONS OF LIE ALGEBRAS
}

\author{
TSUNEO KanNo
}

(Received January 16, 1956)

0. In [3] H. Zassenhaus has given a determination of the irreducible representations of a nilpotent Lie algebra $\mathfrak{L}$ over an algebraically closed field $K$ of characteristic $p>0$ as follows. For each ordered set $\left(\lambda_{1}, \lambda_{2}, \ldots \ldots\right.$, $\lambda_{n}$ ) of elements of $K$, there exists one and only one equivalence class $\{U\}$ of irreducible representations of $\mathfrak{L}$ such that $\lambda_{i}$ is the unique eigenvalue of $U\left(x_{i}\right)$, where $x_{1}, x_{2}, \ldots, x_{i b}$ is a regular base of $\mathfrak{I}$. Recently, in [1], replacing the set of scalars by the set $\left(f_{1}, \ldots, f_{n}\right)$ of irreducible polynomials, C. W. Curtis has proved that for each set $\left(f_{1}, \ldots, f_{n}\right)$ there exists an equivalence class $\{U\}$ of irreducible representations of $\mathfrak{L}$ such that the minimal polynomial of $U\left(x_{i}\right)$ is a power of $f_{i}$, when $K$ is an arbitrary field of characteristic $p>0$. But generally the uniqueness of the existence of the class does not hold in Curtis' case. In this paper we shall give an answer to the problem of the one-to-one crrrespondence in his case and at the same time we consider this problem in the case of soluble Lie algebras over an arbitrary field $K$ of characteritic 0 .

The author wishes to thank Professor T. Tannaka and Dr. C. W. Curtis.

1. We begin with some elementary results. Let $\mathfrak{L}$ be a Lie algebra over an arbitrary field $K$ with a basis $x_{1}, x_{2}, \ldots, x_{n}$, and let $\mathfrak{A}$ be the universal enveloping algebra of $\mathfrak{L}$. If we imbed the vector space $\mathfrak{L}$ into $\mathfrak{A}$, we obtain a basis $x_{1}^{o_{1}} x_{2}^{\rho_{2}} \ldots x_{n}^{o_{n}}, e_{i} \geqq 0$ of $\mathfrak{U}$ over $K$, where $x_{1}^{i} x_{2}^{0} \ldots x_{n}^{0}=1$. Then we have a natural one-to-one correspondence between the representations ${ }^{1)}$ of $\mathfrak{L}$ and those of $\mathfrak{A}$, which is described as follows. To any representation $U$ of $\mathfrak{L}$, we may correspond the representation $U^{\prime}$ of $\mathfrak{A}$ defined by $U^{\prime}\left(x_{i}\right)=U\left(x_{i}\right)$ and $U^{\prime}(1)=E, i=1, \ldots, n$, where $E$ is identity transformation. In the following we identify $U$ with $U^{\prime}$.

Let $U$ be an irreducible representation of $\mathfrak{A}$ with the representation space $\mathfrak{U}$. For any non-zero element $u$ of $\mathfrak{U}$, we have $u U(\mathfrak{H})=\mathfrak{U}$. Let $\Im$ be the right ideal of $\mathfrak{U}$ which consists of elements $a$ such that $u U(a)=0$, then we have the difference group $\mathfrak{A}-\mathfrak{\Im}$ of $\mathfrak{A}$ by the maximal right ideal $\mathfrak{\Im}$ and the right $\mathfrak{A}$-module $\mathfrak{A}-\mathfrak{Y}$ is of finite dimension over $K$. Let us denote by $\mathfrak{N}$ the set of all elements $a \in A$ such as $\mathfrak{N} a \subset \mathfrak{S}$. Then $\mathfrak{N}$ is the largest twosided ideal of $\mathfrak{A}$ contained in $\mathfrak{F}$. We shall call $\mathfrak{A}$ the quotient of $\mathfrak{F}$ relative to $\mathfrak{A}$.

These definitions give us

LEMMA 1. Let $\Im_{i}$ and $\mathfrak{N}_{i}$ be a right ideal of $\mathfrak{A}$ and its quotient relative to $\mathfrak{A}, i=1,2$. If two $\mathfrak{A}$-modules $\mathfrak{A}-\mathfrak{Y}_{1}$ and $\mathfrak{U}-\mathfrak{F}_{2}$ are isomorphic, then $\mathfrak{N}_{1}=\mathfrak{N}_{2}$. Further, if $\mathfrak{\Im}_{i}$ is a two-sided ideal, $i=1,2$, then $\mathfrak{\Im}_{1}=\mathfrak{\Im}_{2}$.

1) In this paper we consider the representations of finite dimension only. 
LEMMA 2. 'If a right ideal $\mathfrak{Y}$ of $\mathfrak{A}$ contains [R, $\mathbb{R}$ ], then $\mathfrak{F}$ is a two-sided ideal and $\mathfrak{A} / \Im$ is commutative ring.

PROOF. Let $x_{1}, x_{2}, \ldots x_{r}, \ldots, x_{n}$ be a base of $\mathfrak{L}$ such that $x_{1}, \ldots, x_{r}$ span $[\mathfrak{R}, \mathfrak{R}]$. We write $\mathfrak{S}=[\mathfrak{Q}, \mathfrak{Q}] \mathfrak{U}$. Every element of $(5$ is expressed in the form $\sum_{i=1}^{r} x_{i} a_{i}$, where $a_{i} \in \mathfrak{A}$. For $j>r, x_{j} \sum_{i=1}^{r} x_{i} \mathfrak{A} \subset \sum_{=1}^{\prime} x_{i} x_{j} A+\sum_{i=1}^{r}\left[x_{j}, x_{i}\right] \mathfrak{A}$

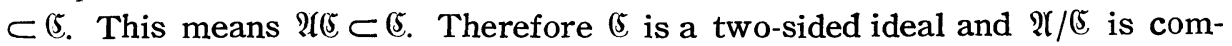
mutative ring. It is easy to see that $\mathfrak{F}$ is a two-sided ideal and $\mathfrak{A} / \mathfrak{F}$ is commutative.

q. e. d.

LEMMA 3. Let $\mathfrak{L}$ be an $n$-dimensional Lie algebra over $K$. If the dimension of $[\mathfrak{L}, \mathfrak{L}]$ is $r$, then the polynomial ring $K\left[X_{1}, \ldots, X_{n-r}\right]$ and the ring $\mathfrak{U} / \mathfrak{S}$ are isomorphic, where (5) is the two-sided ideal [R, $\mathfrak{Q}] \mathfrak{A}$.

Proof. Let $x_{1}, \ldots, x_{r}, \ldots, x_{n}$ be the base of $\mathbb{L}$ such that $x_{1}, \ldots, x_{r}$ span $[\mathcal{L}, \mathfrak{R}]$. Then $a=\sum \alpha_{\rho_{1} \ldots e_{n}} x_{1}^{e_{1}} \ldots x_{n}^{e_{n}}$ is contained in 5 if and only if for every $\alpha_{e_{1} \ldots e_{n}} \neq 0$, there exists the positive integer $s \leqq r$ such that $e_{1}=e_{2}=\ldots=$ $e_{s-1}=0$, and $e_{s} \neq 0$. In fact, take monomials of the form $x_{i}^{e i} \ldots x_{r}^{e} \ldots \ldots x_{n}^{n}$, $i \leqq r$, and suppose that for $i<j \leqq r$ and $s=\sum_{k=i}^{i-1} e_{k} \leqq t-1$, we have straighted the element $x_{j} x_{i}^{e_{i}} \ldots x_{r}^{i} \ldots \ldots x_{n}^{e_{n}}$ into the required canonical form. Then in the case of $s=t$, we obtain $x_{j} x_{i_{i}}^{e} \ldots x_{n}^{e}=x_{i} x_{j} x_{i}^{i-1} \ldots x_{n}^{\rho_{n}}+\sum_{k=1}^{\prime \prime} \gamma_{j i}^{k} x_{i} x_{i}^{e l-1}$ $\ldots x_{n}^{n}$ where $\gamma_{j i}^{k}$ 's are structural constants of $\mathcal{L}$. Now here we may apply the assumption of induction. Since every element of 5 is expressed in the form $\sum_{i=1}^{r} x_{i} \cdot a_{i}, a_{i} \in \mathfrak{A}$, we may prove the necessary condition. The inverse is trivial.

Now let $\phi$ be a homomorphism of $K\left[X_{1}, \ldots, X_{n-r}\right]$ into $\mathfrak{A}-\mathfrak{s}$ defined by $\varphi\left(X_{i}\right)=x_{r+i}+5, i=1, \ldots, n-r$. Then since every non-zero class modulo (5) has such a representative as $\sum \alpha_{e_{r+1} \ldots e_{n}} x_{r+1}^{e_{r+1}} \ldots x_{n}^{e_{n}, \phi}$ is onto-homomorphism. And it is easily seen that if for some $f\left(X_{1}, \ldots, X_{1 b-r}\right) \in K\left[X_{1}, \ldots\right.$, $\left.X_{n-r}\right], \quad \phi\left(f\left(X_{1}, \ldots, X_{n-r}\right)\right) \in\left(5, f\left(X_{1}, \ldots, X_{n-r}\right)=0\right.$. Thus $\phi$ is onto-isomorphism.

q. e. d.

The Corrollary of Theorem 3 and Theorem 5 of [2] have the following consequence.

LEMMA 4. If $\mathfrak{M}$ is a maximal ideal $K\left[X_{1}, \ldots, X_{s}\right]$, there exists a chain $\mathfrak{M}_{1} \subset \mathfrak{M}_{2} \subset \ldots \subset \mathfrak{M}_{s}=\mathfrak{M}$ where $\mathfrak{M}_{i}=\mathfrak{M} \cap K\left[X_{1} \ldots, X_{i}\right]$ is a maximal ideal of $K\left[X_{1}, \ldots, X_{i}\right], i=1.2, \ldots, s$.

We shall call the chain $\mathfrak{M}$-chain associated with the maximal ideal $\mathfrak{M}$.

Now we shall prove the lemma which is obtained from the proof of Theorem 1 of Curtis [1].

LEMMA 5. Let $\mathfrak{L}$ be a nilpotent Lie algebra over an arbitrary field $K$ and $U$ and $V$ be two irreducible representations of $\mathfrak{R}$. If for each $x \in \mathbb{L}, U(x)$ and $V(x)$ have the same eigenvalue $\alpha(x)$, then these two representations are equivalent. 
Proof. Let $\widetilde{K}$ be an algebraic closure of $K$ and let $\widetilde{U}$ and $\widetilde{V}$ be the scalar extensions of $U$ and $V$ from $K$ to $\widetilde{K}$, respectively. Let $m$ and $n$ be the degrees of $U$ and $V$. Then we have a representation $\widetilde{T}(x)=\widetilde{U}(x) \otimes E_{n}$ $-E_{m} \otimes \widetilde{V}(x)^{t}$ where $E_{n}$ and $E_{m}$ are identity matrices of degree $m$ and $n_{\text {r }}$ respectively, and $\widetilde{V}\left(x^{t}\right.$, means the transpose of $\widetilde{V}(x)$. Let $\mathfrak{U}$ and $\mathfrak{B}$ be the representation spaces of $U$ and $V$, then there exist non-zero vectors $u \in \mathfrak{H}_{\tilde{K}^{-}}$

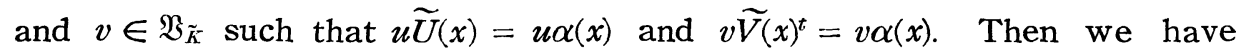
$(u \otimes v) \widetilde{T}(x)=(u \otimes v) \widetilde{U}(x) \otimes E_{n}-(u \otimes v) E_{m} \otimes \widetilde{V}(x)^{t}=u \widetilde{U}(x) \otimes \widetilde{v}-u \otimes\left(v \widetilde{V}(x)^{t}\right)$ $=0$. This means zero is an eigenvalue of $\widetilde{T}(x)$ and $\operatorname{det} \widetilde{T}(x)=0$, for $x \in L_{\tilde{K}}$. Since $\widetilde{K}$ is infinite field, there exists a non-zero vector $\widetilde{w} \in\left(U_{\tilde{K}} \otimes V_{\tilde{K}}\right)=(U$ $\otimes V) \tilde{K}$ such that $\tilde{w} \widetilde{T}(x)=0$ for all $x \in \mathfrak{Q}_{\tilde{K}}$ (cf. Proposition 2 of [1]). If we write $\widetilde{w}=\sum w_{i} \lambda_{i}, \lambda_{i} \in \widetilde{K}$, where $w_{i} \in \mathfrak{U} \otimes \mathfrak{B}$ and $\lambda_{i}$ 's are linearly independent over $K$, we have $\tilde{w} T(x)=\sum w_{i} T(x) \lambda_{i}=0$, for all $x \in \mathfrak{L}$, and hence $w_{i} T(x)$ $=0$.

Thus we may find a non-zero element $w$ of $\mathfrak{U} \otimes \mathfrak{B}$ such that $w T(x)=0$, for all $x \in \mathfrak{R}$. We write $w=\sum\left(u_{i} \otimes v_{j}\right)\left(w_{i j}\right)$ where $u^{\prime}$ s and $v^{\prime}$ s are bases of $\mathfrak{U}$ and $\mathfrak{B}$. The simple calculation yeilds $U(x) \cdot W=W \cdot V(x)$, for all $x \in \mathfrak{L}$, where $W$ is the matrix $\left(w_{i j}\right)$. Since $U$ and $V$ are irreducible, $U$ and $V$ are equivalent.

q. e. d.

2. In this section we shall consider a soluble Lie algebra $\mathfrak{L}$ over an arbitrary field $K$ of characterestic 0 . Let $\mathfrak{F}$ be a maximal right ideal of $\mathfrak{A}$ such that the dimension of $\mathfrak{A}$-module $\mathfrak{A}-\mathfrak{Z}$ is finite. Then, since by Lie's Theorem any irreducible representation of $\mathfrak{L}$ is abelian, $[\mathfrak{L}, \mathfrak{L}] \subset \mathfrak{R} \subset \mathcal{F}$ where $\mathfrak{N}$ is the quotient of $\mathfrak{Y}$ relative to $\mathfrak{A}$. So by Lemma $2 \mathfrak{F}$ is a two-sided ideal and $\mathfrak{A} / \Im$ is a (commutive) field $K^{\prime}$ which is finite (algebraic) extension over $K$. Writing $K^{\prime}=K$ if the irreducible representation $U$ is trivial, we obtain the field $K^{\prime}$ which is uniquely determined by the irreducible representation $U$.

Then we have

Proposition. Let $\mathfrak{Q}$ be a soluble Lie algebra over an arbitrary field $K$ of characteristic 0 , let $D$ be a derivation of $\mathbb{R}$, let $x \rightarrow U(x)$ be an irreducible representation of $\mathfrak{Q}$ with the representation space $\mathfrak{U}$ such that $U(D(\mathfrak{L}))=0$, and let $f(X)$ be an irreducible polynomial in $K^{\prime}[X]$ where $K^{\prime}$ is the field determined by $U$ as mentioned above. Then, there exists exactly one equivalence class $\{W\}$ of irreducible representation of the semi-direct sum $\left.{ }^{2}\right) \mathfrak{L}+K \cdot D$ with the representation space $\mathfrak{Z}$ and a one-to-one linear transformation $S$ of $\mathfrak{U}$ into

2) The semi-direct sum $L+K \cdot D$ is the Lie algebra whose underlying vector space is the direct sum of vector spaces $\mathrm{L}$ and $K \cdot D$, and in which the bracket multiplication is defined by the formula $\left[x+\alpha D, x^{\prime}+\alpha^{\prime} D\right]=\left[x, x^{\prime}\right]+x^{\prime} D(x)-\alpha D\left(x^{\prime}\right), x, x^{\prime} \in \mathrm{L}$, $\alpha, \alpha^{\prime} \in K$. 
$\mathfrak{W}$ such that $U(x) S=S W(x)$ for all $x \in \mathfrak{L}$ and $f(X)$ is an irreducible factor of the minimal polynomial of $W(D)$ in $K^{\prime}[X]$.

PRoof. Let $\mathfrak{A}$ be the universal enveloping algebra of $\mathfrak{L}$. If $x_{1}, x_{2}, \ldots, x_{n}$ is a basis of $\mathfrak{L}$, then the standard monomials $x_{1}^{1} x_{2}^{\ell_{2}} \ldots x_{n}^{\ell_{n}} D^{t}, e_{i} \geqq 0, d \geqq 0$ form a basis of the universal enveloping algebra $\mathfrak{A}^{\prime}$ of $\mathfrak{I}+K \cdot D$. Let $\mathfrak{Y}$ be the maximal right ideal of $\mathfrak{A}$ such that $\mathfrak{A}$-module $\mathfrak{A}-\mathfrak{S}$ induces $U$. We may suppose that $\mathfrak{U}=\mathfrak{A}-\mathfrak{Z}$ and $U(x): u+\mathfrak{S} \rightarrow u x+\mathfrak{Y}$, for $x \in \mathfrak{L}$. Then $\mathfrak{G} \mathfrak{Y}^{\prime}$ is a proper ideal of $\mathfrak{U}^{\prime}$. In fact, every element $a$ of $\mathfrak{Y}^{\prime}$ can be expressed uniquely in the form $a=\sum a_{i} D^{\prime}, a_{i} \in A$ where if $a \in \mathfrak{Y} \cdot \mathfrak{H}^{\prime}$, then $a_{i} \in \mathfrak{Y}$. If $\mathfrak{Y} \mathfrak{U}^{\prime}=$ $\mathfrak{A}^{\prime}$, then the identity 1 is contained in $\mathfrak{F}^{\prime}$ and $1=\sum a_{i} D^{i}, a_{i} \in \mathfrak{\Im}$. Therefore $1=a_{0} \in \mathfrak{Y}$, contrary to the fact that $\mathfrak{Y}$ is proper ideal. From $U(D(\mathbb{Q})$ ) $=0$, we have $D_{\mathfrak{L}}^{\prime} \mathfrak{R} \subset \mathfrak{G}$ and $[\mathfrak{Z}+D, \mathfrak{L}+D] \subset \mathfrak{F}$. By Lemma $2, \mathfrak{Y} \mathfrak{H}$ is a twosided ideal of $\mathfrak{U}^{\prime}$ and ' $\mathfrak{U}{ }^{\prime} / \mathfrak{F} \mathfrak{Y}$ ' is commutative. The mapping $\phi: \sum a_{i} D^{i}+$ $\mathfrak{F U}^{\prime} \rightarrow \varphi\left(\sum a_{i} D^{i}+\mathfrak{G} \mathfrak{Y}^{\prime}\right)=\sum\left(a_{i}+\mathfrak{F}^{\prime}\right) D^{i}$ gives the isomorphism of $\mathfrak{A}^{\prime}-\mathfrak{F H}^{\prime}$ onto $(\mathfrak{A}-\mathfrak{\Im})[D]=K^{\prime}[D]$. Thus we have a one-to-one correspondence between maximal ideals of $\mathfrak{U}^{\prime}$ which contain $\mathfrak{Y} \mathfrak{Y}^{\prime}$ and maximal ideals of $K^{\prime}[D]$. Let $\mathfrak{Y}^{\prime}$ be a maximal ideal of $\mathfrak{A}$ snch that $\phi\left(\mathfrak{Y}^{\prime}\right)=(f(D))$. It is easily seen that the dimension of the $\mathfrak{H}^{\prime}$-module $\mathfrak{H}^{\prime} / \mathfrak{Y}^{\prime}$ over $K$ is finite. Since $\mathfrak{S} \subset \mathfrak{Y}^{\prime} \subset \mathfrak{Y}^{\prime}$, $\mathfrak{Y} \subset \mathfrak{J}^{\prime} \cap \mathfrak{A}$. But $\mathfrak{I}^{\prime} \cap \mathfrak{A}$ is a proper ideal of $\mathfrak{A}$ and $\mathfrak{Y}$ is maximal, $\mathfrak{Y}^{\prime} \cap \mathfrak{A}$ $=\mathfrak{3}$.

Define $\mathfrak{B}=\mathfrak{A}^{\prime} / \mathfrak{Y}^{\prime}$ and $W(x) ; w+\mathfrak{Y}^{\prime} \rightarrow w x+\mathfrak{Y}^{\prime}$. Since $\mathfrak{Y}^{\prime} \cap \mathfrak{A}=\mathfrak{Y}$, the mapping $S: u+\mathfrak{F} \rightarrow u+\mathfrak{Y}^{\prime}$ is a one-to-one linear transformation of $\mathfrak{u}$ into $\mathfrak{B}$ such that $U(x) \cdot S=S \cdot W(x)$, for $x \in \mathbb{R}$.

Since $\mathfrak{Y}^{\prime}$ is the two-sided ideal of $\mathfrak{U}^{\prime}$, the quotient $\mathfrak{N}^{\prime}$ of $\mathfrak{Y}^{\prime}$ relative to $\mathfrak{A}^{\prime}$ coincides with $\Im^{\prime}$. Let $f_{0}(X) \in K[X]$ be the minimal polynomial of $W(D)$. Then $f_{0}(D)$ is the polynomial of the least degree in $K[D] \cap \Im^{\prime}$. Therfore $f_{0}(D)=\phi\left(f_{0}(D)+\mathfrak{Y} \mathfrak{Y}^{\prime}\right) \in(f(D)) \subset K^{\prime}[D]$, and $f(D)$ is an irreducible factor of $f_{0}(D)$ in $K^{\prime}[D]$

q. e. d.

By Lemmas 1,3 and theorem of Lie, we have

THEOREM 1. Let $\mathfrak{Q}$ be a soluble Lie algebra over an arbitrary field $K$ of characteristic 0 . Then there exists a one-to-one correspondence between equivalence classes of irreducible representations of $\mathfrak{Q}$ and maximal ideals of the polynomial ring $K\left[X_{1}, \ldots, X_{s}\right]$ where $s$ is the dimension of $\mathbb{L} /[\mathbb{L}, \mathfrak{Q}]$ over $K$.

Further

THEOREM 1 '. Let $\mathfrak{Q}$ be a soluble Lie algebra over an arbitrary field $K$ of characteristic 0, let $x_{1}, \ldots, x_{s}, \ldots, x_{n}$ be a base of $\mathfrak{Q}$ such that $x_{s+1}, \ldots, x_{n}$ span $[\mathfrak{Q}, \mathfrak{Q}]$, and $f_{i}\left(X_{i}\right)$ be an irreducible polynomial in $K_{i}\left[X_{i}\right]$ defined inductively such as $K_{1}=K$ and $K_{i}=K_{i-1}\left[X_{i-1}\right] /\left(f_{i-1}\left(X_{i-1}\right)\right)$. Then there exists a one-to-one correspondence between equivalence classes $\{U\}$ of irreducible representations of $\mathfrak{R}$ and such sets $\left(f_{1}, \ldots, f_{s}\right)$, and if $U$ corresponds to $\left(f_{1}, \ldots, f_{s}\right), f_{i}(X)$ is an irreducible factor of the minimal polynomial of $U\left(x_{i}\right)$ in $K_{i}[X], i=1, \ldots$ s. 
PRoof. Let $\mathfrak{L}_{i}$ be the ideal of $\mathfrak{Q}$ spanned by $x_{1}, \ldots x_{i}, x_{s+1}, \ldots, x_{n}$. Let $\mathfrak{A}_{i}$ be the universal enveloping algebra of $\mathfrak{L}_{i}, i=1,2, \ldots, s$. Then we may suppose that $\mathfrak{A}_{i} \subset \mathfrak{A}_{i+1}$. By Lemma 3 we have an isomorphism $\varphi$ of $K\left[X_{1}\right.$, $\left.\ldots, X_{s}\right]$ onto $\mathfrak{U}-\left(\mathfrak{s}\right.$ such that $\varphi\left(X_{i}\right)=x_{i}+(5$, where $(5,=[\mathfrak{Q}, \mathfrak{Q}] \mathfrak{U}$.

Let $U$ be an irreducible representation of $\mathfrak{L}$ and let $\mathcal{Z}$ be a maximal right ideal of $\mathfrak{A}$ such that the $\mathfrak{A}$-module $\mathfrak{A}-\mathfrak{Y}$ induces $U$. By Lemmas 1,2 and Lie's theorem, $\Im$ is the two-sided ideal determined uniquely by the equivalence class $\{U\}$ which contains $U$. We write $\mathfrak{M}=\boldsymbol{\phi}^{-1}(\mathfrak{Y})$. Let $\mathfrak{M}_{1} \subset \mathfrak{M}_{2} \subset$ $\ldots \subset \mathfrak{M}_{s}=\mathfrak{M}$ be $\mathfrak{M}$-chain (cf. Lemma 4). Then we obtain a set $\left(f_{1}, \ldots, f_{s}\right)$ as mentioned in the theorem. We prove this by induction. We write $\mathfrak{B}_{t}=$ $\mathfrak{M}_{t-1} \cdot K\left[X_{t}, \ldots, X_{t}\right], t=2,3 \ldots s$. Let $\sigma_{t}$ be the natural isomorphism of $K\left[X_{1}, \ldots, X_{i}\right] / \mathfrak{R}_{t}$ onto $\left(K\left[X_{1}, \ldots, X_{t-1}\right] / \mathfrak{M}_{t-1}\right)\left[X_{t}\right]$ defined by $\sigma_{t}\left(\sum a_{\nu} X_{t}^{\prime}+\mathfrak{P}_{t}\right)$ $=\sum\left(a_{\nu}+\mathfrak{M}_{t-1}\right) X_{i}^{\prime}$, for $a_{\nu} \in K\left[X_{1}, \ldots, X_{t-1}\right], t=2, \ldots, s$. At first we have the irreducible polynomial $f_{1}\left(X_{1}\right) \in K\left[X_{1}\right]$ such that $\left(f_{1}\left(X_{1}\right)\right)=\mathfrak{M}_{1}$, and let $\tau_{1}$ be the identity automorphism of $K_{1}\left[X_{1}\right] /\left(f_{1}\left(X_{1}\right)\right)=K_{2}$. Assume that we have constructed the natural isomorphism $\tau_{i}$ of $K\left[X_{1}, \ldots X_{i}\right] / \Re_{i}$ onto $K_{i}\left[X_{i}\right]$ and the irreducible polynomial $f_{i}\left(X_{i}\right)$ such that $\left.\tau_{i}\left(\mathfrak{M}_{i}\right)=f_{i}\left(X_{i}\right)\right)$ for $i=2, \ldots$, $t-1$. Then, let $\widetilde{\tau}_{t-1}$ be the induced isomorphism of $K\left[X_{1}, \ldots, X_{t-1}\right] / \mathfrak{M}_{t-1}$ onto $K_{t-1}\left[X_{t-1}\right] /\left(f_{t-1}\left(X_{t-1}\right)\right)=K_{t}$, and we have the required isomorphism $\tau_{t}=\widetilde{\tau}_{t-1} \sigma_{t}$ of $K\left[X_{1}, \ldots, X_{t}\right] / \Re_{t}$ onto $K_{t}\left[X_{t}\right]$ and the irreducible polynomial $f_{t}\left(X_{t}\right) \in K_{t}\left[X_{t}\right]$ such that $\tau_{t}\left(\mathfrak{M}_{t}\right)=\left(f_{t}\left(X_{t}\right)\right)$. The set $\left(f_{1}, \ldots, f_{t}\right)$ determined uniquely by $\mathfrak{M}$ is called $\mathfrak{M}$-set associated with $\mathfrak{M}$. If $\bar{f}_{i}(X)=\sum \alpha_{i j} X^{j} \in K[X]$

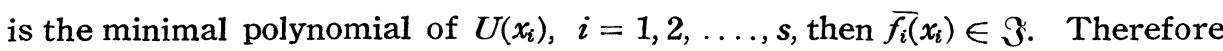
$\varphi^{-1}\left(\overline{f_{i}}\left(X_{i}\right)+\mathfrak{E}\right)=\overline{f_{i}}\left(X_{i}\right) \in \mathfrak{M} \cap K\left[X_{1}, \ldots, X_{i}\right]=\mathfrak{M}_{i}$ and $\tau_{i}\left(\overline{f_{i}}\left(X_{i}\right)+\mathfrak{B}_{i}\right)=\overline{f_{i}}\left(X_{i}\right)$ $\in\left(f_{i}\left(X_{i}\right)\right)$. This means that $f_{i}(X)$ is an irreducible factor of the minimal polynomial of $U\left(x_{i}\right)$ in $K_{i}[X]$.

Conversely, for a given set $\left(f_{1}, \ldots, f_{s}\right)$, there exists only one maximal ideal $\mathfrak{M}$ of $K\left[X_{1}, \ldots, X_{s}\right]$ whose $\mathfrak{M}$-set is the given $\left(f_{1}, \ldots, f_{s}\right)$ (cf. Proof of Proposition 1). Writing $\mathfrak{F}=\phi(\mathfrak{M})$, then we obtain a required equivalence class of the irreducible representations of $\mathfrak{L}$ which is induced by $\mathfrak{A} / \mathfrak{\Im}$. q. e.d.

3. Let $\&$ be a nilpotent Lie algebra over an arbitrary field $K$ of characterstic $\boldsymbol{p}>0$. It is well known that any nilpotent Lie algebra has a regular base $x_{1}, x_{2}, \ldots, x_{n}$ such that, whenever $i<j,\left[x_{i}, x_{j}\right] \in \sum_{k=1}^{i-1} K x_{i k}$. In this section we use a regular base only. There exists a positive integer $r$ such that $y_{i}=x_{i}^{p}$ is contained in the center of the universal enveloping algebra $\mathfrak{A}$ of \&. We write $\mathfrak{B}=K\left[y_{1}, \ldots, y_{n}\right]$ and $\subseteq=\sum_{\left(e_{1}, \ldots, e_{n}\right) \neq 0(\bmod p} p^{r} K x_{1}^{e_{1}} \ldots \ldots x_{n}^{i r}$ where $\left(e_{1}, \ldots, e_{n}\right) \neq 0\left(\bmod p^{r}\right)$ means that there exists $i \leqq n$ such that $e_{i}$ 丰 0 (mod. $\left.p^{r}\right)$. Then we have $\mathfrak{A}=\mathfrak{B}+\mathfrak{S}, \mathfrak{B} \cap \mathfrak{S}=(0)$ and $\mathfrak{B} \mathfrak{S}=\mathfrak{S} \mathfrak{B} \subset \mathfrak{S}$. Every element $a \in \mathfrak{A}$ has its unique expression $a=B(a)+S(a), B(a) \in \mathfrak{B}, S(a) \in \mathfrak{S}$, where $B$ and $S$ are linear transformations of $\mathfrak{A}$ onto $\mathfrak{B}$ and $\mathcal{S}$ respectively. Then we have 
LEMMA 6. For a maximal ideal 5 of $\mathfrak{B}$, there exists a maximal right ideal $\mathfrak{Y}$ of $\mathfrak{A}$ such that $\mathfrak{Z} \cap \mathfrak{B}=\mathfrak{6}$.

Proof. $\left(\mathfrak{S} \mathfrak{A} \neq \mathfrak{A}\right.$. In fact, if $\mathfrak{5} \cdot \mathfrak{A}=\mathfrak{A}, 1=\sum c_{i} a_{i}$ where $c_{i} \in \mathfrak{S}, a_{i} \in \mathfrak{A}$. Then $\sum c_{i} a_{i}=\sum c_{i} B\left(a_{i}\right)+\sum c_{i} S\left(a_{i}\right)=1$. Therefore, $1=\sum c_{i} B\left(a_{i}\right) \in \sqrt{5}$, contrary to the fact that $(5$ is proper ideal of $\mathfrak{B}$. Since the dimension of the $\mathfrak{A}$-module $\mathfrak{A}-\mathfrak{C} \mathfrak{A}$ is finite, there exists a maximal right ideal $\mathfrak{F}$ such that $\Im \supset(5$. On the other hand, since $\mathfrak{F}$ does not contain $1, \mathfrak{F} \cap \mathfrak{B}$ is proper and $\Im \cap \mathfrak{B}=(5$.

q.e.d.

LEMMA 7. Let (5, be a maximal ideal of $\mathfrak{B}$. If there are two maximal right ideals $\Im_{1}$ and $\Im_{2}$ of $\mathfrak{A}$ such that $\Im_{i} \cap \mathfrak{B}=\mathfrak{G}, i=1,2$, then two $\mathfrak{A}$-modules $\mathfrak{A}-\mathfrak{\Im}_{1}$ and $\mathfrak{A}-\mathfrak{Y}_{2}$ are isomorphic.

Proof. Let $U_{i}$ be irreducible representations of $\mathfrak{L}$ induced by $\mathfrak{A}$-module $\mathfrak{A}-\mathfrak{Y}_{i}$, for $i=1,2$. Since $(\mathfrak{B}+\mathfrak{F})-\mathfrak{Y}_{i} \cong \mathfrak{B}-\mathfrak{C}, i=1,2$, the two representations $U_{i}(\mathfrak{B}) \mid\left(\mathfrak{B}+\mathfrak{\Im}_{1}\right)-\mathfrak{\Im}_{1}$ and $U_{2}(\mathfrak{B}) \mid\left(\mathfrak{B}+\mathfrak{Y}_{\mathfrak{3}}\right)-\mathfrak{\Im}_{3}$ of $\mathfrak{B}$ are equivalent, where the notation $\mid$ means restriction of $U_{i}$ to the submodule $\left(\mathfrak{B}+\Im_{i}\right)-\Im_{i}$ of $\mathfrak{A}-\mathfrak{Y}_{i}, i=1,2$. Let $\widetilde{K}$ be the algebraic closure of $K$. Then $\widetilde{U}_{1}(\mathfrak{B}) \mid((\mathfrak{B}+$ $\left.\left.\mathfrak{\Im}_{1}\right)-\mathfrak{Y}_{1}\right)_{\tilde{K}}$ is equivalent to $\widetilde{U}_{2}(\mathfrak{B}) \mid\left(\left(\mathfrak{B}+\mathfrak{F}_{2}\right)-\mathfrak{\Im}_{2}\right) \tilde{K}_{\text {K }}$ where $\widetilde{U}_{i}$ and $\left(\left(\mathfrak{B}+\mathfrak{\Im}_{i}\right)\right.$ $\left.-\Im_{i}\right)_{\tilde{K}}$ mean the scalar extensions of $U_{i}$ and $\left(\left(\mathfrak{B}+\mathfrak{\Im}_{i}\right)-\mathfrak{\Im}_{i}\right)$ from $K$ to $\widetilde{K}$. There exists indecomposable component $\mathfrak{B}_{i}$ of $\left(\left(\mathfrak{B}+\mathfrak{\Im}_{i}\right)-\mathfrak{\Im}_{i}\right) \tilde{K}, i=1$, 2 , such that $\widetilde{U}_{1}(\mathfrak{B}) \mid \mathfrak{B}_{1}$ is equivalent to $\widetilde{U}_{4}(\mathfrak{B}) \mid \mathfrak{B}_{2}$. Then, for $y \in \mathfrak{B}$ we have the same unique eigenvalue $\alpha(y)$ of $U_{i}(y) \mid \mathfrak{B}_{i} i=1,2$. (cf. Theorem 1 of [1]). The module $\mathfrak{B}_{i}^{\prime}=\mathfrak{B}_{i} \widetilde{U_{i}}(\mathfrak{H})$ is an invariant submodule of $\left(\mathfrak{A}-\Im_{i}\right) \tilde{\kappa}, \quad i=1,2$. Since $\widetilde{U_{i}}(\mathfrak{B})$ is central there exists a positive integer $R$ such that $1 \mathfrak{B}_{i} \widetilde{U_{i}}(\mathfrak{U})\left(\widetilde{U_{i}}(y)-\alpha(y)\right)^{R}=\mathfrak{B}_{\mathfrak{\ell}}$ $\left(\widetilde{U}_{i}(y)-\alpha(y)\right)^{R} U(\mathfrak{H})=0, i=1,2$. Let $W_{i}(\mathfrak{H})$ be an irreducible constituent of $\widetilde{U_{i}}(\mathfrak{H}) \mid \mathfrak{B}_{i}^{\prime}$ with the representation space $\mathfrak{B}_{i}$ which is a submodule of $\mathfrak{B}_{i}^{\prime}$. Then $\mathfrak{B}_{i}\left(\widetilde{U}_{i}\left(x_{j}\right)-\alpha\left(y_{j}\right)^{\frac{1}{p^{\prime \prime}}}\right)^{p^{\prime} R}=\mathfrak{W}_{i}\left(U_{i}\left(y_{j}\right)-\alpha\left(y_{j}\right)\right)^{R}=0$, for $i=1,2$, and $j=1$, $2, \ldots, n$. This means that $\alpha\left(y_{i}\right)^{\frac{1}{p^{r}}}$ is the same unique eigenvalue of $\widetilde{U}_{i}\left(x_{j}\right) \mid \mathfrak{W}_{i}, i=1,2, j=1,2, \ldots, n$. Since $x_{1}, \ldots, x_{n}$ is a regular base of $\mathfrak{L}$, by Zassenhaus' theorem, $\widetilde{U}_{1}(\mathfrak{H}) \mid \mathfrak{W}_{1}$ and $\widetilde{U}_{2}(\mathfrak{H}) \mid \mathfrak{M}_{2}$, are equivalent. Therefore for $x$ $\in \mathfrak{Q}, \widetilde{U_{1}}(x)$ and $\widetilde{U_{:}}(x)$ have the same eigenvalue. The application of the Lemma 5 yields that these two irreducible representations are equivalent. q. e.d.

LEMMA 8. Let $\mathfrak{F}_{\imath}$ be a mamimal right ideal of $\mathfrak{A}$ such that the $\mathfrak{A}$-module $\mathfrak{A}-\mathfrak{F}_{\mathrm{t}}$ is of finite dimention, let $\mathfrak{S}$ be a maximal ideal of $\mathfrak{B}$ which contains $\mathfrak{I}_{1} \cap \mathfrak{B}$ and let $\mathfrak{\Im}_{2}$ be a maximal right ideal of $\mathfrak{A}$ which contains $(5$. Then two $\mathfrak{U}$-modules $\mathfrak{A}-\mathfrak{Z}_{1}$ and $\mathfrak{A}-\mathfrak{Y}_{2}$ are isomorphic. above.

REMARK. For the right ideal $\Im_{1}$ there exist $\left(\varsigma\right.$ and $\mathfrak{\Im}_{2}$ as mentioned

PRoof. Since $\left(\mathfrak{B}+\mathfrak{F}_{1}\right)-\mathfrak{\Im}_{1} \cong \mathfrak{B}-\left(\mathfrak{\Im}_{1} \cap \mathfrak{B}\right)$ and $\left(\mathfrak{B}+\mathfrak{\Im}_{2}\right)-\mathfrak{\Im}_{2} \cong \mathfrak{B}-(5$ the representation $U_{1}(\mathfrak{B}) \mid\left(\mathfrak{B}+\mathfrak{\Im}_{1}\right)-\mathfrak{Y}_{1}$ has an irreducible constituent which 
is equivalent to $U_{2}(\mathfrak{B}) \mid\left(\mathfrak{B}+\mathfrak{\Im}_{2}\right)-\mathfrak{\Im}_{2}$. Since irreducible constituents of indecomposable "component of $U_{1}(\mathfrak{B}) \mid\left(\mathfrak{B}+\mathfrak{\Im}_{1}\right)-\mathfrak{F}_{1}$ are equivalent; there exists a submodule $\mathfrak{E}$ of $\left(\mathfrak{B}+\mathfrak{Z}_{1}\right)-\mathfrak{Z}_{1}$ such that $U_{1}(\mathfrak{B}) \mid \mathfrak{F}$ is equivalent to $U_{2}(\mathfrak{B}) \mid(\mathfrak{B}$ $\left.+\mathfrak{Y}_{2}\right)-\mathfrak{3}_{2}$. The argument which we have developed in the proof of Lemma 6 gives Lemma 7 .

q. e.d.

If $\mathfrak{F}_{1}$ and $\mathfrak{\Im}_{2}$ are right ideals of $\mathfrak{A}$ such that $\mathfrak{A}-\mathfrak{\Im}_{1} \cong \mathfrak{A}-\mathfrak{\Im}_{2}$, we have $\mathfrak{N}_{1}=\mathfrak{N}_{2}$ where $\mathfrak{N}_{i}$ is the quotient of $\mathfrak{\Im}_{i}$ relative to $\mathfrak{A}$ (cf. Lemma 1). Since $\mathfrak{N}_{i}$ is the largest two-sided ideal of $\mathfrak{A}$ contained in $\mathfrak{\Im}_{i}, \mathfrak{\Im}_{i} \cap \mathfrak{B} \subset\left(\mathfrak{\Im}_{i} \cap \mathfrak{B}\right) \cdot \mathfrak{A} \subset \mathfrak{N}_{i}$ and $\mathfrak{Y}_{i} \cap \mathfrak{B} \subset \mathfrak{A}_{i} \cap \mathfrak{B}$. But since $\mathfrak{R}_{i} \subset \mathfrak{Y}_{i}$ and $\mathfrak{R}_{1}=\mathfrak{A}_{\mathfrak{2}}$, we have $\mathfrak{Y}_{1} \cap \mathfrak{B}=$ $\mathfrak{F}_{2} \cap \mathfrak{B}$. Therefore applying the Lemma 8 , we have

LEMMA 9. If $\mathfrak{Y}$ is a maximal right ideal of $\mathfrak{A}$ such that the dimension of $\mathfrak{A}$-module $\mathfrak{A}-\mathfrak{Y}$ is finite, $\mathfrak{Y} \cap \mathfrak{B}$ is a maximal ideal of $\mathfrak{B}$.

The Lemmas 7 and 9 have the following consequence.

THEOREm 2. Let $\mathfrak{\&}$ be a n-dimensional nilpotent Lie algebra over an arbitrary field of characterstic $p>0$. There exists one-to-one correspondence between equivalence classes of irreducible representations of $\mathbb{Q}$ and maximal ideals of polynomial ring $K\left[X_{1}, X_{2}, \ldots, X_{n}\right]$.

\section{Further}

THEOREM 2'. Let $\mathbb{Q}$ be a nilpotent Lie algebra over an arbitrary field $K$ of characteristic $p>0$, let $x_{1}, x_{2}, \ldots, x_{n}$ be a regular base of $\mathbb{Q}$ and let $f_{i}\left(X_{i}\right)$ be an irreducible polynomial in $K_{i}\left[X_{i}\right]$ defined inductively such as $K_{1}=K$ and $K_{i}=K_{i-1}\left[X_{i-1}\right] /\left(f_{i-1}\left(X_{i-1}\right)\right)$ for $i=2, \ldots, n$. Then there exists a one-to-one correspondence between equivalence classes $\{U\}$ of irreducible representations of $\mathfrak{L}$ and such sets $\left(f_{1}, \ldots, f_{s}\right)$ and if $U$ corresponds to $\left(f_{1}, \ldots, f_{s}\right), f_{i}(X)$ is an irreducible factor of the minimal polynomial of $U\left(x_{i}^{p r}\right)$ in $K_{i}[X]$ where $r$ is a positive integer such that $x_{i}^{p^{r}}$ is central in the enveloping algebra of $\mathbb{L}$.

Proof. By Lemmas 6 and 7 we may consider maximal ideals of $\mathfrak{B}$. The argument which has been developed in the proof of the Theorem $1^{\prime}$ runs in this case, too.

q.e.d.

Here is an example of two irreducible representations of Lie algebra which are not equivalent and correspond to the same set of irreducible polynomials in the sense of Curtis' Theorem 2 of [1].

ExAmple. Let $K$ be a field which does not contain $\sqrt{2}$, and let $\mathfrak{L}=K x_{1}$ $+K x_{2}$ be a two-dimensional abelian Lie algebra over $K$. Then two represen-

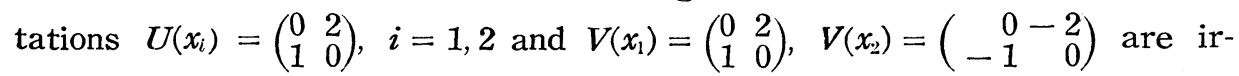
reducible and not equivalent. The irreducible polynomial $X^{2}-2$ is the minimal polynomials of $U\left(x_{i}\right)$ and $V\left(x_{i}\right), i=1,2$.

\section{REFERENCES}

[1] C. W. CuRTIS, A note on the representations of nipotent Lie algebras, Proc. Amer. 
Math. Soc., 5(1954) 813-824.

[2] O. Goldman, Hilbert rings and the Hilbert Nullstellensatz, Niath. Zeit. 54(1951) 136-140.

[3] H Zassenhaus, Úber Liescine Ringe mit Primzahlcharakteristik, Abh. Math. Sem. Hansischen Univ., 13(1940) 1-100.

MATHEMATICAL INSTITUTE, TÔHOKU UNIVERSITY 